\title{
Editorial
}

\section{A Snapshot on Nuclear Cardiac Imaging Khalil, $M$.}

\author{
Department of Physics, Faculty of Science, Helwan University.
}

There is no doubt that nuclear medicine scanning devices are essential tool in the diagnostic process and patient workup. Nuclear medicine hardware can be classified into two major categories namely imaging and non-imaging systems. Both should be integrated in a very systematic manner during daily practice of many nuclear medicine procedures and examinations. The down side of this view is that a little error in one of those imaging chain could propagate towards a large error in the final image interpretation and diagnostic outcome. Quality assurance and quality control systems should be seriously in place so that the maximum benefit of nuclear medicine exam is attained and proper clinical service is provided.

\section{Myocardial perfusion SPECT is commonly performed using dual-head gamma cameras based on $\mathrm{NaI}(\mathrm{Tl})$ scintillation detectors with photomultiplier tube array and high-resolution parallel-}

Corresponding Authors: Khalil, M. hole collimators. However, limitations of these SPECT systems- namely their low count sensitivity and poor spatial resolution - mean that it is necessary to administer activities in the range of 296$444 \mathrm{MBq}$ for the first radiopharmaceutical injection for cardiac perfusion study and three times as much for the second injection on the same day. In most cases both rest and stress cardiac study are carried out in two separate days due to the low image quality and presence of attenuation artifacts especially in absence of attenuation correction (AC) (e.g., Gd153 line source, CT or supine and prone imaging). The total injected activities in such procedure (summing both rest and stress injected activities) are in the range of $1.184-1.776 \mathrm{MBq}$ leading to patient effective dose of $13.5 \mathrm{mSv}$ per patient with acquisitions lasting approximately for $15 \mathrm{~min}$, and overall study times of up to $4 \mathrm{~h}$ per patient in the case of one-day stress/rest protocols ${ }^{(\mathbf{1})}$.

E-mail: magdy_khalil@hotmail.com. 
Advances in SPECT imaging hardware, software, and radiopharmaceutical are significantly improving the non-invasive evaluation of myocardial perfusion and function. In contrast to traditional dualheaded, sodium iodide crystal and photomultiplier cameras with mechanical collimators, new SPECT camera designs utilize novel, collimators, and solid-state detectors (e. CZT) that convert photons directly to electrical signals. These cameras simultaneously collect data from as many small detectors that narrowly focused on the heart ${ }^{(2)}$.

New noise handling and resolution recovery reconstruction software interprets emitted counts more efficiently and thus more effectively discriminates between useful signals and noise. Hence, shorter acquisition times and/or lower tracer doses produce higher quality SPECT images than conventionally implemented. Hybrid SPECT/CT cameras utilize transmission computed tomographic (CT) scans for AC, and offer the additional clinical advantages of evaluating coronary calcium scoring, myocardial anatomy (including noninvasive CT angiography), myocardial function, and myocardial perfusion in a single imaging procedure ${ }^{(\mathbf{2 . 3})}$.

Novel collimator designs, such as multipinhole and locally focusing collimators arranged in geometries that are optimized for cardiac imaging have been implemented to reduce imaging time and radiation dose. These new collimators have been coupled with solid state photon detectors to improve image quality and reduce scanner size. The new SPECT scanners demonstrate up to a 7-fold increase in photon sensitivity and up to 2 times improvement in image resolution ${ }^{[3]}$.

Recently, Lecchi et al evaluated the impact of reduced count statistics on the fully quantitative estimation of summedrest scores (SRS), summed-stress scores (SSS), end-systolic volume (ESV), enddiastolic volume (EDV), and ejection fraction (EF). They demonstrated no significant differences even with $1 / 4$ dose scans (the scans with 8-s acquisition time per projection) for normal patients and no differences for $1 / 2$ simulated dose for obese patients. They use objective quantitative analysis and appropriate simulated protocols, allowing for the evaluation of the effect of the progressively reduced dose in the same patient ${ }^{(4)}$.

Advantages of their study included objective assessment of myocardial perfusion quantitative parameters. Moreover, they studied overweight and normal weight groups separately, which 
Allowed them to evaluate the appropriateness of reducing the radiation dose or time separately in patients with different categories of weight.

Another study showed that using innovative gamma cameras, collimators and software, as well as, unless otherwise indicated, stress-first imaging protocols has become possible to reduce significantly the effective dose in a high percentage of patients, even when X-ray CT scanning is performed for attenuation correction $^{(\mathbf{1})}$.

The development of high-efficiency cardiac dedicated scanners equipped with cadmium-zinc-telluride (CZT) detectors has been paralleled by the development of single photon emission computed tomography (SPECT) systems combined with X-ray computed tomography (CT) scanners, mostly employed for attenuation correction $(\mathrm{AC})$ of the emission data.

Thus, although the advanced technologies may allow reductions in patient doses, the new combined scanners, due to the CT component, might actually lead to an increase in radiation exposure ${ }^{[1,3]}$. The iterative reconstruction and resolution recovery algorithms (IRR) were introduced into clinical practice primarily to reduce acquisition times, since fast imaging provides immediate benefits in terms of patient throughput and patient comfort. However, equivalent dose reduction is also possible by performing standard time acquisitions with lower injected activities (5).

Using the IRR algorithm Astonish ${ }^{\mathrm{TM}}$ (Philips), the possibility of decreasing the dose to $25 \%$ of the reference value was evaluated in the context of a two-day imaging protocol, without $\mathrm{AC}(3 \mathrm{mSv}$ for a full examination). The authors found that decreasing radiopharmaceutical activity to $25 \%$ of the reference value found practicable for normal-weight patients, while an activity reduction down to $50 \%$ was suggested to be more appropriate for obese and overweight patients [4]. Other studies investigated the possibility of halving the dose to patients using the reduced acquisition time to simulate an equivalent reduction of the injected activity.

With full-time acquisitions (15 min), a $50 \%$ dose reduction seems to be the limit for IRR algorithms. With today's SPECT/CT systems the use of a stress-first protocol can conveniently be performed ${ }^{(5)}$. If two-thirds of patients were considered definitively normal after the stress study, there would be no need to perform the rest study in these patients. 
In such a situation, patient dose would decrease by $76 \%$, but the CT dose has to be added, resulting in an overall dose reduction of about $35 \%(\mathbf{1 , 4})$.

Two systems (Discovery NM 530c, General Electric and D-SPECT, Spectrum Dynamics) having the same configuration as multiple CZT detectors, but coupled with different high-sensitivity collimators (multi-pinhole vs high-sensitivity parallelhole collimators, respectively) and IRR algorithms, have been introduced on the market. A comparative study, performed with an anthropomorphic phantom mimicking the thorax of a normal adult, has shown relatively similar physical performance of the two CZT cameras, even though the corresponding images of the left ventricle insert were significantly different, revealing a smaller wall thickness with the D-SPECT than with the Discovery NM 530c $\quad(13.6 \pm 0.7 \quad$ vs $19.6 \pm 1.3 \mathrm{~mm}$, respectively). It is unlikely, however, that such differences have a significant impact on diagnostic accuracy (6).

While solid-state cardiac cameras improve spatial and energy resolution and allow for markedly reduced SPECT acquisition times and/or injected radioactive dose, they have some distinct drawbacks and
Short comings including high cost and lack of attenuation correction. Cardiofocused collimation, advantageous to increase depth-dependent resolution and myocardial count density, accentuates diaphragmatic attenuation and scatter from sub-diaphragmatic structures ${ }^{(7)}$.

Moreover, obese and large patients cannot be accommodated in the solid-state camera gantries. Patient motion can be neither detected nor corrected since data are acquired simultaneously with an arc of solid-state detectors around the chest; no temporally dependent "rotating" projection images are obtained. In contrast, traditional sodium iodide SPECT cameras provide rotating projection images to allow technologists and physicians to detect and correct patient motion and to accurately detect the position of soft tissue attenuators and to anticipate associated artifacts ${ }^{(8)}$.

Very large patients are easily accommodated. Low-dose x-ray attenuation correction is widely available. Also, relatively inexpensive low-count density software is provided by many vendors, allowing shorter SPECT acquisition times and reduced injected activity approaching that achievable with solid-state cameras. 
In conclusion, there is a lot of innovations are still under extensive efforts in nuclear cardiac imaging including new detector systems, reconstruction methodologies, dose reductions, faster acquisitions

\section{REFERENCES:}

\section{Lecchi M, Malaspina S, Scabbio}

C, Gaudieri V, Del Sole A. Myocardial perfusion scintigraphy dosimetry: optimal use of SPECT and SPECT/CT technologies in stress-first imaging protocol. Clin. Transl. Imaging. 4:491498; 2016.

2. Garcia EV. Physical attributes, limitations, and future potential for PET and SPECT. J. Nucl. Cardiol.19 Suppl 1:S19-29; 2011.

\section{Slomka PJ, Berman DS, Germano}

G. New cardiac cameras: single-photon emission CT and PET. Semin. Nucl. Med. 44:232-251; 2014.

\section{Lecchi M, Martinelli I, Zoccarato}

$\boldsymbol{O}$, et al. Comparative analysis of full-time, half-time, and quarter-time myocardial ECG-gated SPECT quantification in modules and more integral data processing of the hybrid SEPCT/CT imaging systems. This would definitely impact patient throughput, diagnostic quality, as well patient management.

normal-weight and overweight patients. J. Nucl. Cardiol. 24:876-887; 2016.

5. Abdollahi H, Shiri I, Salimi Y, et al. Radiation dose in cardiac SPECT/CT: An estimation of SSDE and effective dose. Eur. J. Radiol. 85:2257-2261; 2016.

6. Zoccarato O, Lizio D, Savi A, et al. Comparative analysis of cadmiumzincum-telluride cameras dedicated to myocardial perfusion SPECT: A phantom study. J. Nucl. Cardiol. 23:885-893; 2016.

7. Lee WW. Recent Advances in Nuclear Cardiology. Nucl. Med. Mol. Imaging. 50:196-206; 2016.

8. DePuey EG. Traditional gamma cameras are preferred. J. Nucl. Cardiol. 23:795-802; 2016. 